\title{
DIFFRAKTION, INDIVIDUATION, SPEKULATION
}

\author{
von JULIA BEE / JENNIFER EICKELMANN / KATRIN KÖPPERT
}

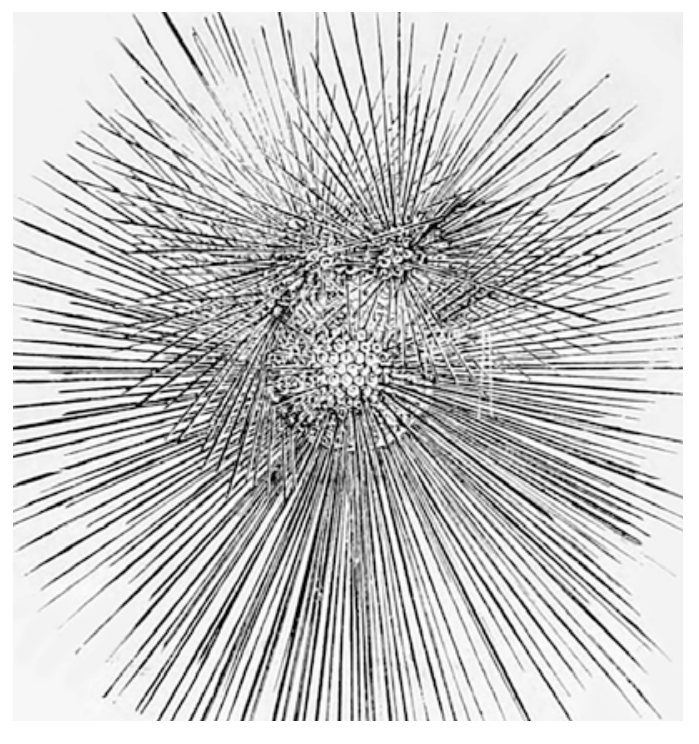

DIFFRAKTION, ODER:

WIE DIE BEUGUNG DER METHODENFRAGE

DIE FRAGE DER METHODE VERÄNDERT

Feministische Verantwortlichkeit erfordert ein Wissen, das auf Resonanz und nicht auf Dichotomie eingestellt ist. ${ }^{1}$

Das ökonomische wie politische Begehren² nach insbesondere disziplinär rückgebundenen methodischen Verfahrensweisen wirft Fragen nach Anerkennungsordnungen auf. Kaum abzustreiten ist, dass die aktuelle Situation disziplinäre Fachverständnisse offenkundig werden lässt, die mit Grenzziehungen und (De-)Legitimationen wissenschaftlicher Praxis einhergehen. Mein Beitrag zu diesem Text positioniert sich als transdisziplinäre und gender-/queertheoretische Perspektive zwischen Medienwissenschaft und Soziologie. Er folgt der Method(ologi)e der Diffraktion, auch wenn die Produktivität dieses Ansatzes wohl nur angedeutet werden kann. Ausgehend von der Annahme, dass auf Grenzen angewiesene Eindeutigkeiten auf komplexen Interferenzen beruhen, wird der Versuch unternommen, Prozesse der Grenzziehung zum Gegenstand zu machen. ${ }^{3}$ Das scheint nicht zuletzt deswegen produktiv, da die Sozialwissenschaften nicht selten als Abgrenzungsfolie medienwissenschaftlicher Vergewisserungen angeführt werden. ${ }^{4}$ So können zum einen Grenzziehungsprozesse thematisiert und zum anderen Vorschläge für andere Zukünfte gemacht werden.

\section{Das Subjekt des Methodendiskurses}

In den Sozialwissenschaften gehören <Methodenfragen> längst zu einem zentralen Bereich. Methodenschulen erfüllen die Funktion, in Lehrbüchern präsentierte Verfahrensweisen innerhalb sozialwissenschaftlicher Disziplinen und durchaus auch darüber hinaus zu verbreiten - als $<$ Methodenexporte>.

Die Frage, wie Theorie zur Anwendung gebracht werden kann und soll, ist dabei bereits dermaßen spezialisiert, dass bestimmte epistemologische Grundannahmen zwar unweigerlich mitlaufen, aber in manchen Methodendiskursen 
nur selten zur Diskussion stehen. Auf entsprechende Situierungen wird leider vielerorts verzichtet, was es deutlich zu problematisieren gilt. Aber es wäre zu kurz gegriffen zu behaupten, in diesem Rahmen würden zuvorderst instrumentelle Fixierungen vorgenommen. ${ }^{5}$ Innerhalb soziologischer Methodendebatten, insbesondere im Kontext der qualitativen Forschung, gelten Abweichungen von <idealtypischen> Verfahrensweisen, je nach Situation und Material, sehr wohl als legitim, zum Teil sogar als unausweichlich. Diese methodischen Spielräume werden in sozialwissenschaftlichen Methodendiskursen zuvorderst unter Rekurs auf die Figur der $<$ Reflexivität thematisiert, die auch in der Medienwissenschaft relevant ist und von unterschiedlichen Stimmen aufgerufen wird. Mit Blick auf die Funktion des Begriffs in der Soziologie ist jedoch festzustellen, dass die Spiegelung von Setzungen und Festschreibungen im Kontext empirischer Forschungsdesigns unter Rekurs auf die Figur der $<$ Reflexivität $>$ ebendiese zwar idealerweise sichtbar macht, aber eben auch selten Anderes evoziert. Vor dem Hintergrund repräsentationskritischer Ansätze im Allgemeinen und im Zuge einer Methodologie der Diffraktion im Besonderen lässt sich die Figur der Reflexion bzw. Reflexivität also insbesondere deswegen problematisieren, da sie nicht etwa an anderen Zukünften, sondern an der Spiegelung des Immergleichen orientiert ist. Mit der Metaphorik der Diffraktion hat Donna J. Haraway eine Denkweise vorgeschlagen, welche in Abgrenzung zur Metaphorik des Spiegels die Dinge anders zur Erscheinung bringt. Diese Denkweise wäre eine gewinnbringende Alternative für Methodendebatten sowohl in der Medienwissenschaft als auch in den Sozialwissenschaften, denn: $\ll[\ldots]$ reflexivity is not enough to produce self-visibility». ${ }^{6} \mathrm{Um}$ diese Setzungen zu problematisieren, reicht eine Unmittelbarkeitsskepsis, wie sie in medienwissenschaftlichen Diskursen fest etabliert ist, nicht aus, da hier gerade keine Unmittelbarkeit behauptet wird. Es geht um ein anderes Problem: In der empirischen Sozialforschung wird wissenschaftliche Praxis zumeist als Zusammenkunft eines forschenden Subjekts und eines zu erforschenden Objekts konzeptualisiert, wobei das forschende Subjekt sich im Prozess als Problem erweist. Vermeintliche Defizite wie Willkürlichkeit, Affizierbarkeit und zum Teil auch Unkontrollierbarkeit werden (im deutschsprachigen Diskurs) häufig als Problem der Vermittlung begriffen, wobei ebenjene Vermittlung auf das involvierte Subjekt zurückgeführt wird. Mithilfe von Methoden wird in die Zusammenkunft von forschendem Subjekt und zu erforschendem Objekt eine (wie auch immer geartete und zum Teil auch nur rhetorische) Rationalität implementiert: Verfahrensspezifikationen regulieren das forschende Subjekt und damit die wissenschaftliche Praxis selbst. ${ }^{7}$ Jenseits der Frage, ob methodische Regulierungen abzulehnen oder zu verteidigen sind, sind Weiterentwicklungen der Debatte insbesondere dann zu erwarten, wenn die Frage der Methoden nicht etwa zur Entscheidung zwingt, ihnen entweder grundsätzlich zu misstrauen ${ }^{8}$ oder sie als «Problemlöser» zu überschätzen, ${ }^{9}$ sondern alternative Methodenverständnisse entwickelt werden - und das meint auch «die Imagination der Zukünftigkeit von Vergangenheit in der Gegenwart», wie Katrin Köppert in ihrem Beitrag betont. Die dualismus- wie subjektkritischen Einsätze der feministischen Theorie versprechen alternative Sichtweisen, die Auswege aus einer verengten Methodendebatte bieten können. Die Beugung eben jenes Dualismus im Sinne der von Haraway vorgeschlagenen Heuristik der Diffraktion kann so beispielsweise zutage fördern, dass wir es hier zwar mit gegenläufigen Bewertungen der regulativen Kraft von Methoden zu tun haben, diese allerdings gleichermaßen an der Re-Souveränisierung von Subjektivität arbeiten. Während eine grundsätzliche Methodenskepsis an der Re-Souveränisierung von Subjektivität mithilfe der Befreiung von Methoden arbeitet, 
leistet der Diskurs, der Methoden als notwendiges Regulativ begreift, der Re-Souveränisierung von Subjektivität mithilfe von Methoden Vorschub. Letztlich sind beide auf einer «vehement verteidigten Subjektposition ${ }^{10}$ basierenden Positionen miteinander verschränkt. Denn die Komplexität der Methodenfrage wird zugunsten der Gegenüberstellungen von Zwang/Freiheit bzw. Problem/Lösung verengt, die sich nicht zuletzt in Subjektivitätsvorstellungen materialisiert.

So betrachtet lässt sich auch die politische Dimension der Debatte besser darstellen: Denn Methodendiskurse dienen auch einer Art der Profilierung im wissenschaftlichen Feld, die das Phantasma souveräner Verfügungsgewalt reproduziert: entweder über sich selbst bzw. über Theorien (jenseits methodischer Zwänge ${ }^{11}$ ) oder eben über Methoden (im Sinne der <reflektierten> Anwendung von Verfahrensweisen und damit «prozeduraler Legitimation ${ }^{12}$ ).

Entsprechend scheint die Frage vielversprechend: Was, wenn das Methodenproblem nicht als genuines Problem der Verfahrensweisen diskutiert wird, sondern als Subjektivitätsproblem?

\section{Verbindungen eingehen!}

Die Dezentrierung des Subjekts kann als einer der wichtigsten Anker gendertheoretischer Argumentationen in Anlehnung an beispielsweise Michel Foucaults Subjektkritik benannt werden. ${ }^{13}$ Es gilt daher einmal mehr, die Produktivität unterschiedlicher Ansätze im Feld der Gender Studies herauszustellen und für unterschiedliche Disziplinen fruchtbar zu machen, bieten sie doch gerade Anregungen für eine wissenschaftliche Praxis jenseits der Zentrierung des (als souverän imaginierten) Subjekts und jenseits der Aktualisierung altbekannter wie machtvoller Dualismen.

Eine alternative Denkweise wäre es, Methoden nicht zuvorderst als Regulativ zu begreifen (und damit weder als Zwangsjacke noch als Problemlösung), sondern als produktives Moment. Wie Julia Bee in ihrem Beitrag schreibt, geht es darum, Bewegungen zu initiieren, Denkweisen zu hinterfragen, Gewohnheiten zu irritieren. Mit Haraway und unter Rekurs auf das Konzept des situierten Wissens gerät wissenschaftliche Praxis als produktives Involviertsein in den Blick, die Generativität ebenjener Praxis kann nicht in Subjekten und <ihren> Objekten aufgehen, sondern stellt unterschiedliche Diskurse, Wissenschaftspraktiken, Technologien und Körper in ihrem wechselseitigen Werden heraus. ${ }^{14}$ Vereindeutigungen und Grenzziehungen geraten so als Machtverhältnisse in den Blick, denen nicht etwas entgegen-, sondern neben die etwas gesetzt werden muss. Letztlich scheint es produktiv, Phantasmen der Souveränität und Verfügungsgewalt samt ihrer legitimierenden Kraft in wissenschaftlichen Diskursen als solche sichtbar zu machen und Alternativen zu entwickeln. Dies kann nur mit partialen Zugängen zwischen Epistemologie und Ontologie gelingen, die wissenschaftliche Praxis als prekäre, d.h. grundlegend relationale, Praxis begreifen. Damit wäre ein Weg eingeschlagen, der zu inter- und transdisziplinären Randgängen einlädt, ließe sich dabei doch besonders gut über Zukünfte jenseits souveräner Subjektivität diskutieren.

Denkt man wissenschaftliche Praxis jenseits starrer disziplinärer Grenzen, erscheint eine Vielfalt an Verbindungsmöglichkeiten, die es erlauben, Geschichten gemeinsamen Werdens zu entwickeln, über die wir letztlich eben nicht vollständig verfügen. Das ist im Übrigen auch der Grund, warum die Gender Studies ohne disziplinäre Vereindeutigungen auskommen, auch wenn die förderpolitischen Bedingungen diesen Vorzug meistens (noch?) nicht anerkennen.

JENNIFER EICKELMANN 


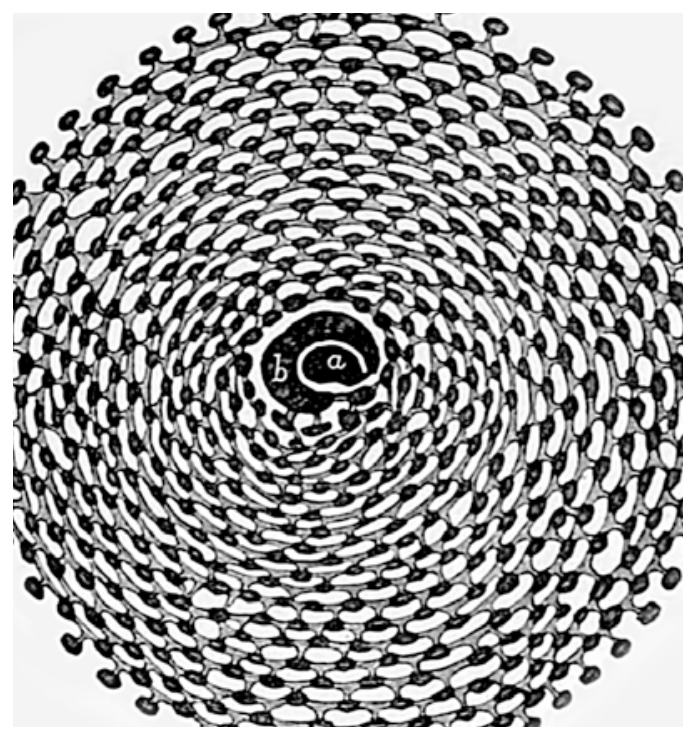

\section{INDIVIDUATION, ODER: VOM METHODENPROBLEM ZUM METHODENEXPERIMENT}

Ich beginne mit zwei Fragen, die möglicherweise naiv klingen mögen. Dabei möchte ich daran erinnern, dass wir es in der Diskussion um Methoden mit einer komplexen Gemengelage an Anrufungen und Diskursen, also machtvollen Gefügen zu tun haben.

Wie kam es eigentlich dazu, dass das Nichtanwenden einer Methode als Freiheit gilt? Und warum nutzen wir diesen Raum hier nicht, um uns gegenseitig unsere Methoden vorzustellen und neue zu erproben? Die erste Frage kann ich in diesem Rahmen nicht vollständig diskursanalytisch zurückverfolgen, aber ich vermute, es hat etwas mit der Wahrnehmung der Äußerlichkeit von Methoden gegenüber den Medienwissenschaften und dem Ergebnis der eigenen Forschung zu tun. Methoden werden aus anderen Fachdisziplinen oder von Förderinstitutionen als aufgenötigt empfunden. Dies hat etwas damit zu tun, wie auch in dieser Debatte häufiger erwähnt wurde, dass wir eigene Methoden haben, die nicht als solche anerkannt wurden. Die Anrufung, Methoden anzuwenden, wird infolgedessen als Einschränkung einer Freiheit der Wissensproduktion empfunden. Diese gilt es heute wieder stark gegen den Umbau hin zur neoliberalen Universität einerseits und andererseits gegen rechte Bewegungen und ihre Antigenderismen und Antiintellektualismen zu verteidigen. Die Angst vor der Disziplinierung der Methoden gilt allerdings nur, wenn man Methoden als Korsett oder als eine Art Automatismus versteht, als Protokoll, welches man zur Fragestellung dazuaddiert, etwas Sekundäres oder dem Ergebnis (sogar der Welt!) Übergestülptes.

Seit einigen Jahren und durch den Einfluss Karen Barads und des New Materialism werden Wissen und Apparate der Wissensproduktion zusammengedacht. Dies betrifft auch eine prekär werdende Grenze zwischen Subjekt und Objekt der Wissensproduktion. ${ }^{15}$ Forscher_innen haben ihre eigene Subjektivität schon früher in die Analyse eingetragen - so wie dies Valerie Walkerdine und Vertreter_innen der Cultural Studies auf faszinierende und produktive Weise in den r $980 e r$ Jahren durch die Analyse ihrer Klassen- und Geschlechterposition praktiziert haben. ${ }^{16}$ Subjektivität schreibt sich aber nicht nur in Ergebnisse ein: Forschungs- und Subjektivierungsprozesse beeinflussen sich wechselseitig. ${ }^{17}$ Diese Überlegungen ermöglichen eine Situierung, indem sie der Methode eine Agency zusprechen, die weder automatisch noch von ihr abgelöst ist. Methoden verkörpern nämlich genuine Probleme der Medialität der Wissensproduktion. Methoden sind Operationen, die die Praxis der Forschung privilegieren. ${ }^{18}$ Sich auf ein Denken durch Methoden einzulassen, heißt auch, vom Ergebnis zur Prozessualität überzugehen. Es trägt die Immanenz eines Denkens durch Operationen und Techniken ein. Methoden können so Strategien der Entessenzialisierung sein. Methoden wären daher vor allem eine Möglichkeit, Wissens- und Subjektproduktion zu reflektieren oder sogar - wenn man einer distanzierten Reflexion kritisch gegenübersteht - zu intensivieren. ${ }^{19}$ 
Ich plädiere daher dafür, Methoden nicht als Korsett einer einmalig etablierten Anzahl von Operationen zu sehen, die sich immer wieder abspielen lassen. Ganz im Gegenteil, mit dem Methodendenken ${ }^{20}$ wird das Privileg, sich unsichtbar zu machen, in Frage gestellt.

Wie Jennifer Eickelmann in diesem Text schreibt, sollten Methoden daher auch wissenschaftshistorisch in ihren Kontexten gesehen werden. Unter anderem aus der Soziologie, ${ }^{21}$ von der man sich ja als Medienwissenschaft emanzipieren wollte, und aus der Ethnologie wissen wir, dass Methoden kontextsensibel sind. Gegenstand und Methode stehen in einem engen Wechselverhältnis, sodass sie ein Gefüge bilden. Das ist schon das Prinzip der grounded theory. Wenn wir offen gegenüber diesen seriellen wechselseitigen Operationen der Übersetzung sind, dann können wir uns noch stärker von einem fixierten und gegenständlichen Wissensbegriff lösen und Wissen selbst als situierte Prozessualität verstehen. Wir können uns nicht auf einen Punkt außerhalb der Methode zurückziehen, an dem es keine Verfahren, sondern nur Ergebnisse gibt. Methoden schreiben sich in das ein, was wir als Ergebnis in Forschungsberichten oder in Texten artikulieren. Und noch stärker formuliert: Sie sind selbst Quelle neuen Wissens, Vehikel der Spekulation, anstatt neutrales Drittes. ${ }^{22}$

In Gefüge des Zuschauens habe ich das Anfertigen von Collagen als Methode der Rezeptionsforschung genutzt. ${ }^{23}$ Ich habe die Collagen nicht als wahrhaftigere Zugänge zu einer Wirklichkeit verstanden, sondern als Produzentinnen und Agentinnen, die spezifische Formen von Rezeptionserfahrungen erst durch ihre spezielle Weise der Medialität hervorgebracht haben. Sie haben den Rezipient_innen und auch mir eine andere Weise der Artikulation angeboten. Durch die Methode der Bildforschung wurde deutlich, dass mit anderen Methoden ein anderes Wissen hervorgebracht wird. Die Methode situierte das Wissen. Immer wieder wurde ich gefragt, ob ich denn nun wolle, dass alle <meine> Methode (die im Übrigen aus der Soziologie Helmut Bremers und Christel Teiwes-Küglers stammte und von Stephan Trinkaus und Gerko Egert in das weitere Feld der Bildtheorie übertragen wurde) anwenden sollten. ${ }^{24}$ Diese Frage hat mich stets gewundert. Warum sollte eine Methode unendlich viele andere mögliche Verfahren ersetzen oder standardisieren, anstatt neue Methoden zu inspirieren und deren Erprobung zu ermutigen? Erfinden wir doch weitere Methoden für kommende Untersuchungen. Für mich war das Verfahren von Collagen und Interviews ein ermöglichendes Gefüge, anhand dessen ich zeigen konnte, wie Wissen als spezifisch medialisiertes artikuliert wird (anstatt es nur anders <auszudrücken>). Und dass das Wissen und die Affektivität der Rezipient_innen diesem partikularen Methodengefüge nicht vorausgeht. Natürlich bringt dieser empirische Methodeneinsatz eine enorme Verantwortung mit sich. Ich wurde «Fürsprecherin ${ }^{25}$ eines Rezeptionswissens der Teilnehmenden und die Collagen wurden ihrerseits zu meinen Fürsprecherinnen. Nicht eine spricht für die andere. Vielmehr entsteht eine individuierende $\ll$ Serie $»^{26}$ aus Subjektivierungen und Medialisierungen, nicht aus Objekten. Aber ist dies nicht in anderen Forschungsprozessen auch so? Jeder Forschungsprozess stellt ein spezifisches Gefüge dar. Die Entwicklung, Durchführung, stetige Modifikation und Diffraktion ermöglicht uns, nicht nur unseren Standpunkt wahrzunehmen, sondern ihn auch zu produzieren. Diese Standpunkt- und Wissensproduktion beschreibt die Medialität der Methode. Die feministische Wissenschaftsforschung hat seit Jahrzehnten darauf hingewiesen. Umso erstaunlicher ist es, dass sie bis jetzt noch keinen Eingang in die Methodendiskussion hier gefunden hat. Denn wir wenden als Wissensproduzent_innen immer eine Methode an, so wie wir uns niemals außerhalb der Produktion von Geschlecht und Geschichte befinden. ${ }^{27}$

Wie Gilles Deleuze und Félix Guattari euphorisch schreiben: «Tausend kleine Geschlechter». ${ }^{\mathbf{2}}$ 
Und damit: Tausend kleine Methoden. Deleuze und Guattari sehen Differenzierung, etwa in Geschlechter, als produktives Vehikel des Werdens und als Frage der Techniken (Wie etwa ein Hund werden? Wie ein Wal?). Diese Techniken sind Wissensformen im Sinne Karen Barads. ${ }^{29}$ Ich würde mit Gilbert Simondon hinzufügen, dass sie bewegliche Ontogenesen sind, ${ }^{30}$ die Dinge in Bewegung bringen, Denkstrukturen und Gewohnheiten hinterfragen, neue Beziehungen knüpfen, die Menschen und Akteur_innen einbeziehen. Jeder Forschungsprozess wäre auch immer eine gleichzeitige Entwicklung von Methoden und Individuationen. Methoden sind keine Instrumente, um zum Ergebnis zu kommen, sie sind kein abgegrenztes Drittes zwischen Welt und Wissen über Welt, Methoden bilden ein Milieu der Techniken und damit neue Weisen, sich mit der Welt und denen zu verbinden, die Wissen wollen (also auch begehren).

Kann es also sein, dass die berechtigte Warnung vor einem Methodenhype (angesichts der befürchteten Fremdbestimmung durch die Ökonomisierung des Wissens) eigentlich auch eine Angst vor der Kreativität der Methoden mitschleift? Vor der Veränderung, die damit eintritt, wenn ich Bilder, Filme, Schreibpraktiken und andere Techniken als gleichberechtigte Akteur_innen der Vermittlung betrachte? Oder was passiert, wenn ich die Techniken und das Wissen von Künsten ernst nehme, als mögliche Partner_innen in der Produktion von Wissen? ${ }^{31}$ Und ist es nicht vielleicht auch eine Angst vor der Kontingenz von Wissen, dass es eine Pluralität medialer Verfahren und damit Techniken gibt, die Wissen ganz <anders〉 produzieren, um eine Idee von Kathrin Busch zu verwenden? ${ }^{32}$ Ich denke, auf diesem Weg gibt es viele notwendig anstehende kritische Interventionen und neue Probleme.

Methoden sind gerade keine Fertigpakete, obwohl sie häufig so gelehrt werden. Gleichwohl müssen sie, um als Verfahren zu bestehen und Wissen zu produzieren, eine innere Konsistenz aufweisen. Sie können in ihrer Mannigfaltigkeit die Anwendbarkeitszumutungen der Neoliberalisierung der Universität ebenso in Frage stellen wie das disziplinierende System der Standardisierung von Wissenschaftsprozessen. Dies heißt nicht, dass sie die Intersubjektivität und Objektivität von Wissenschaft unterlaufen. Wie Donna J. Haraway argumentiert, ist Objektivität nicht das Gegenteil von Subjektivität. ${ }^{33}$

Wenn wir die Materialität und Medialität von Apparaten beschreiben, warum tun wir dies so ungerne mit den Prozessen und Praktiken, die diese überhaupt erst herstellen oder neu herstellen, umarbeiten etc.? Darauf ist ja nicht nur die ANT geeicht, sondern vor allem feministische STS. Was ist etwa mit Filmen - sind sie nicht auch, z. B. im Bereich der Visuellen Anthropologie - eine Methode, eine spezifische Existenzweise? Sie sind Prozess der Wissensproduktion und Gegenstand zugleich. Wie es schon aus den interventionistischen und aktivistischen Verfahren des cinéma vérité bekannt ist, verändern sie die Wirklichkeit, die sie erforschen. Sie ermöglichen aber auch durch kollaborative Prozesse neue Existenzen und neue Selbstbezüge, indem ein Filmdreh zu einer Weise der Subjektivierung statt der Objektivierung werden kann. Auch hier sind Machtverhältnisse immanent.

Der Rückbezug auf das eigene Handeln ermöglicht in der Methode auch eine Weise der (temporären, situierten) Subjektivierung. Diese trägt die Spezifität und damit Materialität und Medialität des Wissens - seiner Produktion, aber auch seiner Präsentation -, etwa als Film, Fotostrecke, Essay, in das Ergebnis ein.

Ein experimenteller Dialog über Methoden schließt nicht neue Kämpfe um Deutungshoheiten aus, wie sie Wissenschaft von jeher prägen. Aber was ist die Alternative? Wir haben keinen neutralen Punkt, von dem aus wir keine Methoden anwenden können. Als Medienwissenschaftler_innen sind wir ja eigentlich gewohnt, genau diese Hierarchisierungen und die Unsichtbarmachung von Konstruktionsprozessen von 
Ideen (und übrigens auch von allen Apparaturen, Körpern und Waren) zu hinterfragen. Aus einem philosophischen (und keinesfalls ökonomistischen) Pragmatismus heraus möchte ich etwas zugleich sehr Einfaches und sehr Komplexes vorschlagen: Lasst uns eine Vielzahl an Methoden ausprobieren, modifizieren, austauschen, aber auch verwerfen, kritisieren und neu erfinden. Lasst uns Räume etablieren, in denen Experimente möglich werden und Methoden nutzen, um Wissenschaft und Welt immer wieder zu befragen und diese dabei affirmativ zu vervielfältigen.

JULIA BEE

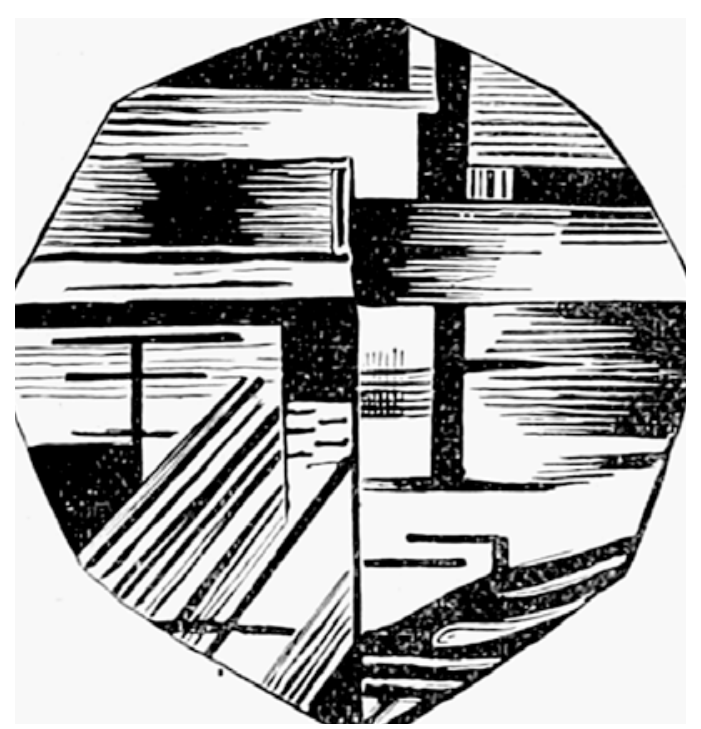

\section{SPEKULATION, ODER: METHOD COMES IN FORM OF STORY TELLING ${ }^{34}$}

Die Aufforderung für dieses kleine Fragment hier lautet: situiertes Schreiben nicht nur als Konzept und Kriterium betrachten, sondern als Form. Der Form halber: Im selben ZfM-Heft, in dem die neuerliche Methodendebatte der Medienwissenschaften angestoßen wurde, befindet sich ein Beitrag, der - da er nicht Teil des Debattenteils ist - als Referenztext für alle bisherigen Folgebeiträge herausgefallen ist. ${ }^{35}$ Zufall? Oder symptomatisch für einen Diskurs, der lieber über Methode im hierfür vorgesehenen Kasten nachdenkt, als mit ihr zu denken, als mit der Methode des Umwegs zu denken? Lesen auf Umwegen. Der Umständlichkeit halber. Umstände machend. Eine feministische Spaßverderberin ${ }^{36} \mathrm{zu}$ sein, ist kein Selbstzweck, keine «<Protestmethodik»», ${ }^{37}$ sondern der ernsthafte Versuch, Fürsprecherin einer Disziplin zu sein, die nicht zum Zwecke der Selbstvergewisserung «Kittler» sagen muss, um Medienwissenschaft zu meinen. Also lese ich auf Umwegen und kann mich überhaupt nur von dort aus ins Verhältnis setzen zu dem, was innerhalb der deutschsprachigen GfMedienwissenschaft so dringend unter dem Aspekt Methode zu diskutieren notwendig scheint. Witzigerweise erscheint an diesem Ort - wie auch bei Jennifer Eickelmann in diesem Text - das, was im Initialtext der Debatte als Problem nahezu heraufbeschworen wird, als fast nicht mehr erwähnenswerte Selbstverständlichkeit. Ja, Medientheorie bedient sich wissenschaftlicher Praktiken, die mit «unterschiedlichen Techniken des Selbst und der Organisation von Wissen, Arbeitsabläufen und Werkzeugen» verbunden sind, schreiben Naomie Gramlich und Annika Haas in «Situiertes Schreiben mit Haraway, Cixous und Grauen Quellen» und ergänzen, dass diese Praktiken im Zusammenhang «mit wechselnden ökonomischen Bedingungen und sich ändernden sozialen Gefügen» erfolgen. ${ }^{38}$ Fertig. Aus.

Methode, die bei den beiden Autorinnen als Ausgangspunkt unmissverständlich gesetzt und lediglich hinsichtlich der (ästhetischen) Form von Situierung diskutiert wird, wird bei Christoph Engemann, Till A. Heilmann und Florian Sprenger wieder zum phantasmatischen Szenario. But why? Was ist die Funktion dessen, Methode wieder zum Problem zu machen? ${ }^{39}$ Was ist die Operation, Methode als Trugbild am 
Horizont einer Medienwissenschaft im vermeintlichen Umbruch aufscheinen zu lassen? Geht es hier wirklich um eine Kritik an der Neoliberalisierung von Universität, die uns ins Korsett Methode zwängt bzw. die Verengung auf empirische Verfahrensweisen in digitalen Zeiten beschert? Man müsste hier im Anschluss an Foucault in die kritische Analyse der Problemstellung einsteigen, also in die Auseinandersetzung mit dem Prozess der Problematisierung, der Aufschluss über die aktuell wirksamen gesellschaftlichen Konstruktionsweisen geben kann. ${ }^{40}$ Aber das ist hier ja kein Raum für Diskursanalyse, sondern eine Debatte. Außerdem spekuliere ich lieber. Und zwar der Form halber. Denn lässt sich nicht vielleicht in der Form der Spekulation eher noch eine kritische, situierte Medienwissenschaft herstellen als in der Anrufung eines Problems?

Die Spekulation geht so: Ich schätze mal, dass mit der Problemstellung Methode ein gewisses Privileg fortgeschrieben werden soll. Nämlich sich leisten zu können, in den Raum zu stellen, auf epistemologische Grundlagen verzichten zu können, auf Quellen, auf das Archiv. ${ }^{41}$ Ich schätze mal, dass eine solche Haltung nur aufgrund des Wissens entstehen kann, dass die Archive gefüllt sind, dass sie jederzeit und aufgrund von Privilegierung, was den Zugang zu Stellen, Ressourcen und Quellen angeht, gefüllt werden können und dass sie einen etwas angehen.

Was aber, wenn dir dieses Archiv nie zugesprochen wurde oder wenn es zwar von dir handelt, aber nur in Form einer Nummer, einer Ware, eines Objekts? Saidiya Hartman schreibt vor dem Hintergrund der transgenerationellen Erfahrung des transatlantischen Sklav_innenhandels, dass, wenn das Archiv dein Todesurteil ist, es zweifellos unmöglich ist, auf es zurückzugreifen, um etwas über dich und deine (Medien-)Geschichte zu erfahren. ${ }^{42}$ Was machst du dann? Was wird aus der Methode, wenn sich das Problem Empirie für dich erst einmal nicht stellt, weil sie entweder immer schon auf das «Verfahren des Fabulierens» ${ }^{43}$ anderer zurückgeht oder als Erfahrungswissen des Selbst im Archiv eine Leerstelle darstellt? Fragst du dann immer noch danach, ob es Methoden braucht? Oder bleibt dir nur Methode, um nicht länger Forschungsgegenstand zu sein, sondern in der Praxis wissenschaftlicher Verfahren Teil eines Prozesses zu werden, der Subjektivierungen erlaubt, wie es Julia Bee in diesem Text schreibt. Aus Perspektive der Marginalisierung ist Methode kein Problem, sondern eine Notwendigkeit, sich subjektivieren zu können.

Doch wie finden marginalisierte Menschen Zugang zu einer Welt, die sich immer schon als falsch erwiesen hat? ${ }^{44}$ Mittels welcher Methoden, anhand welcher Subjektbegriffe? Und was bedeutet dies im Umkehrschluss für diejenigen, denen das Privileg zufällt, in der Welt zu leben, die die Welt lebt? ${ }^{45}$

Hartman fragt: $\ll[\mathrm{H}]$ ow does one rewrite the chronicle of a death foretold and anticipated, as a collective biography of dead subjects, as a counter-history of the human, as the practice of freedom?», ${ }^{\mathbf{4 6}}$ und beantwortet ihre Frage mit dem (Schreib-)Verfahren der kritischen Fabulation. Damit stellt sie die Unmöglichkeit, eine Geschichte erzählen zu können, in den Mittelpunkt eines Schreibens, das den verunmöglichten Geschichten näherkommen will: «I intended both to tell an impossible story and to amplify the impossibility of its telling. ${ }^{\mathbf{4 7}}$ Die Unzulänglichkeit des Schreibens verankert die Methode der kritischen Fabulation. Sie bindet die Methode an das Scheitern. Sie zwingt eine_n, sich immer wieder neu zu fragen, was hätte gewesen sein können, welche Freiheiten hätten gelebt werden können. Damit fällt das forschende Subjekt nicht aus dem Prozess, sondern es wird Modus des Konjunktivs, Spekulation sozusagen. Und damit entzieht sich Methode der Anspruchshaltung, etwas «durch medienhistorische Genealogien rekonstruieren $\gg^{\mathbf{4}}$ zu können. Stattdessen bliebe (Medien-)Wissenschaft darauf verpflichtet, etwas zu imaginieren, das nicht 
verifiziert werden kann. ${ }^{49} \ll[\mathrm{T}]$ he refusal to fill in the gaps and provide closure, is a requirement of this method», ebenso wie der Imperativ all das zu sagen, «which resists being said»..$^{50}$ Die Verweigerung, (Medien-)Wissenschaft als Verfahren der Repräsentation ${ }^{51}$ zu begreifen, rüttelt an den Fundamenten einer westeuropäischen Epistemologie, an deren Anfang das rational denkende Subjekt steht. Aber nichts ist durch diese Verweigerung verloren. Im Gegenteil: «All of [the lost] dreams survive, even when they are rendered imperceptible as such. $\gg^{\mathbf{5}}$ Die Frage ist nun, ob wir nicht lieber das Lamentieren sein lassen sollten, zugunsten eines kritischen Fabulierens, das dabei hilft, die aufgrund von Kolonialismus, Rassismus und Heterosexismus verlorenen Freiheitsträume in der Gegenwart zu imaginieren. Denn auch das - die Imagination der Zukünftigkeit von Vergangenheit in der Gegenwart - ist Aufgabe von Medienwissenschaft. Es wird nur viel zu selten noch als Methode praktiziert bzw. expliziert.

KATRIN KÖPPERT
1 Donna J. Haraway: Situiertes Wissen. Die Wissenschaftsfrage im Feminismus und das Privileg einer partialen Perspektive, in: Elvira Schleich (Hg.): Vermittelte Weiblichkeit. Feministische Wissenschafts- und Gesellschaftstheorie, Hamburg 1996, 217-248.

2 Stichwort ‘Transferuniversität).

3 Donna J. Haraway: The Promises of Monsters: A Regenerative Politics for Inappropriate/d Others, in: Lawrence Grossberg, Cary Nelson, Paula A. Treichler (Hg.): Cultural Studies, London 1992, 295-337; Astrid DeuberMankowsky: Diffraktion statt Reflexion, in: Zeitschrift für Medienwissenschaft, Nr. 4, 2011, 83-91; Karen Barad: Diffracting Diffraction: Cutting TogetherApart, in: parallax, Jg. 20, Nr. 3 , 2014, 168-187.

4 Auch weil sie, als Erfahrungswissenschaften verstanden, verallgemeinernd als das Andere des "Bücherwissens" diskursiviert werden, vgl. Christoph Engemann, Till A. Heilmann, Florian Sprenger: Wege und Ziele. Die unstete Methode der Medienwissenschaft, in: Zeitschrift für Medienwissenschaft, Nr. 20, 2019, 151-161, hier 153 .

5 Vgl. Engemann, Heilmann, Sprenger: Wege und Ziele, $159 \mathrm{f}$.

6 Donna J. Haraway: Modest_ Witness@Second_Millennium.Female-Man`_Meets_OncoMouse ${ }^{T M}$. Feminism and Technoscience, New York, London 1997, 268. Vgl. auch Deuber-Mankowsky: Diffraktion statt Reflexion.

7 Vgl. Reiner Keller, Angelika Poferl: Soziologische Wissenskulturen zwischen individualisierter Inspiration und prozeduraler Legitimation. Zur Entwicklung qualitativer und interpretativer Sozialforschung in der deutschen und französischen Soziologie seit den 196oer Jahren, in: Forum Qualitative Sozialforschung, Jg.17, Nr. 1, 2016, www.qualitativeresearch.net/index.php/fqs/rt/printer Friendly/2419/3942 (12.2.2020).

8 So hat Patrick Vonderau das Misstrauen gegenüber Methoden, wie es im Beitrag von Engemann, Heilmann, Sprenger als medienwissenschaftliche Vergewisserung zu Tage tritt, herausgearbeitet, vgl. ders.: Methode als wissenschaftssoziales Problem, in:
Zeitschrift für Medienwissenschaft, Nr. 21, 165-168, hier $16_{5}$.

9 Vgl. beispielhaft Stephanie Bethmann: Methoden als Problemlöser. Wegweiser für die qualitative Forschungspraxis, Weinheim 2019.

10 Vonderau: Methode als wissenschaftssoziales Problem, 168.

11 Interessanterweise grenzt sich die deutschsprachige Soziologie, die in erster Linie an einer "prozeduralen Legitimation" orientiert ist, zum Teil scharf von der französischsprachigen und deren Ausrichtung an einer «individualisierten Inspiration» ab, vgl. Keller, Poferl: Soziologische Wissenskulturen.

12 Ebd.

13 Vgl. Michel Foucault: Gespräch zwischen Michel Foucault und Studenten. Jenseits von Gut und Böse, in: ders.: Von der Subversion des Wissens, hg. v. Walter Seitter, Regensburg 1974, 110-127; Judith Butler: Kontingente Grundlagen: Der Feminismus und die Frage der ‘Postmoderne», in: Seyla Benhabib u. a.: Der Streit um Differenz. Feminismus und Postmoderne in der Gegenwart, Frankfurt/M. 1995, 31-58.

14 Vgl. auch Naomie Gramlich, Annika Haas: Situiertes Schreiben mit Haraway, Cixous und Grauen Quellen, in: Zeitschrift für Medienwissenschaft, Nr. 20, 2019, 39-52.

15 Karen Barad: Meeting the Universe Halfway, Durham 2007; Corinna Barth u. a.: Geschlechter Interferenzen. Wissensformen - Subjektivierungsweisen - Materialisierungen, Münster 2013.

16 Valerie Walkerdine: Subjektivität, Feminismus, Psychoanalyse, hg. v. Brigitte Hipfl u. Matthias Marschik, Wien 2011. Siehe auch aktuell für das Experiment der De/Subjektivierung im wissenschaftlichen Text: Jason Pine: The Alchemy of Meth. A Decomposition, Minneapolis 2019.

17 Die Methode, durch die Narration von Wissen gleichzeitig situierende und subjektivierende Effekte zu forcieren, zieht sich durch das Werk von Saidiya Hartman, vgl. zur Methode der kritischen Fabulation Katrin Köppert in diesem Text und aktuell Hartman: Wayward Lives, Beautiful Experiments. Intimate Histories of Social Upheaval, New York, London 2019. 
18 Erin Manning: Against Method, in: dies.: The Minor Gesture, Durham 2016, 26-45. Manning ist "gegen Methode» und für Techniken. Mein Verständnis von Methode hingegen entspricht eher ihrem Begriff von Technik. Der Einfachheit halber beziehe ich mich aber hier auf den Begriff Methode.

19 Gilles Deleuze: Differenz und Wiederholung, München 2007, 271-279. Deleuze sieht seine "Methode der Dramatisierung" als Intensivierung von Differenzen, die Individuationen erzeugen. Siehe auch: Die Methode der Dramatisierung, in: ders.: Die einsame Insel. Texte und Gespräche 1953-1974, hg. v. David Lapoujade, Frankfurt/M. 2003, 139-170.

$20 \mathrm{Im}$ Sinne eines Denkens durch Methoden hindurch, ein Denken, in das sich die Techniken der Methode einschreiben.

21 Dies trifft sicher nicht für die ganze Soziologie zu, sondern für gesellschaftlicher Emanzipation verpflichtete Strömungen der Soziologie. Vgl. zur Debatte um eine kritische Soziologie: Geoffroy de Lagasnerie: Denken in einer schlechten Welt, Berlin 2018. Wobei Lagasnerie vorschnell Empirie als unkritisch gegenüber einer Soziologie der Konzepte darstellt.

22 Ich vereinfache mit der anachronistischen Begriffswahl hier bewusst.

23 Julia Bee: Gefüge des Zuschauens. Begehren, Macht und Differenz in Film- und Fernsehwahrnehmung, Bielefeld 2018.

24 Helmut Bremer, Christel Teiwes-Kügler: Die Gruppenwerkstatt. Ein mehrstufiges Verfahren zur vertiefenden Exploration von Mentalitäten und Milieus, in: Heiko Geiling (Hg.): Probleme sozialer Integration. agis-Forschungen zum gesellschaftlichen Strukturwandel, Münster 2003, 207-236; Gerko Egert u. a.: Praktiken der Nichtmännlichkeit - PrekärWerden Männlicher Herrschaft im ländlichen Raum Brandenburg, in: Alexandra Manske, Katharina Pühl (Hg.): Prekarisierung zwischen Anomie und Normalisierung. Geschlechtertheoretische Bestimmungen, Münster 2010, 186-209; Gerko Egert, Stephan Trinkaus: Visualität als relationale Praxis, Vortrag im Rahmen des Workshops «(An)
Erkennen von prekären Leben. Methodologische Verknüpfungen von praxeologischen und queeren Forschungsstrategien", 13.-14.9.2010, Universität zu Köln.

25 Gilles Deleuze: Die Fürsprecher, in: ders.: Unterhandlungen. 1972-1990, Frankfurt/M. 1993, 175-196.

26 Deleuze: Fürsprecher, 181. 27 Vieles an der Debatte über Methoden erinnert mich daher an Debatten um Gender Studies vor vielen Jahren: Methode wird gleichsam als äußerer Zwang artikuliert, so wie einige Wissenschaftler_innen meinen, Gender sei ihrem eigentlichen Denken äußerlich. Ganz ähnlich kann im Forschungsprozess der Eindruck entstehen, die etablierten Methoden seien universell.

28 Gilles Deleuze, Félix Guattari: Tausend Plateaus. Kapitalismus und Schizophrenie 2, Berlin 1992, 291.

29 Barad: Meeting the Universe Halfway.

30 Gilbert Simondon: Das Individuum und seine Genese. Einleitung, in: Claudia Blümle, Armin Schäfer (Hg.): Struktur, Figur, Kontur. Abstraktion in Kunst und Lebenswissenschaften, Zürich 2007, 29-45; ders.: L'Individuation psychique et collective, Paris 2007. Vgl. auch Erin Manning: Engenderings: Gender, Politics, Individuation, in: dies.: Politics of Touch. Sense, Movement, Sovereignty, Minneapolis, London 2008, 84-109.

31 Künste können auch Methoden im Sinne von Techniken entwickeln. Dies wird in den Debatten um künstlerische Forschung häufig umgekehrt diskutiert und gerade im Hinblick auf PhD-Programme, die künstlerische Forschung verwissenschaftlichen. Dabei würde es gerade nicht darum gehen, künstlerische Forschung entsprechend den Wissenschaften zu standardisieren, sondern als Feld der spekulativen Methodenentwicklung im Spannungsfeld von Künsten und Medien zu verstehen.

32 Kathrin Busch (Hg.): Anderes Wissen. Kunstformen der Theorie, München 2016

33 Haraway: Situiertes Wissen. 34 In Anlehnung an Leanne Simpson: Dancing on Our Turtle's Back, Winnipeg, Manitoba 2011.
35 Gramlich, Haas: Situiertes Schreiben.

36 Sara Ahmed: Feministisch leben! Manifest für Spaßuerderberinnen, Münster 2017.

37 Engemann, Heilmann, Sprenger: Wege und Ziele, 155.

38 Gramlich, Haas: Situiertes Schreiben, 39.

39 Michel Foucault beschäftigt sich in seinen späten Vorträgen und Schriften mit dem Problem der Problematisierung und verweist auf die gesellschaftlichen Konstruktionsweisen von Problemen, die gegebenenfalls zu hinterfragen und in die gegebenenfalls zu intervenieren ist. Michel Foucault: Diskurs und Wahrheit. Die Problematisierung der Parrhesia, Berlin 1996, 128.

40 Vgl. auch Ulrike Klöppel: Foucaults Konzept der Problematisierungsweise und die Analyse diskursiver Transformationen, in: Achim Landwehr (Hg.): Diskursiver Wandel, Wiesbaden 2010, $255-263$.

41 Ich möchte mich herzlich bei Anja Sunhyun Michaelsen, Ana de Almeida und Melanie Konrad für den fruchtbaren Austausch zu Archiv und Archivtheorie im Rahmen des Workshops «Family Frames. Perspektiven auf Intimität und Familiarität in fotografischen Medien" (8.11.2019, tfm, Universität Wien) bedanken.

42 Saidiya Hartman: Venus in Two Acts, in: Small Axe, Jg. 12, Nr. 2, 2008, 1-14, hier 2; vgl. auch dies.: Lose Your Mother. A Journey Along the Atlantic Slave Route, New York 2007.

43 Achille Mbembe: Kritik der schwarzen Vernunft, Frankfurt/M. 2015, 31.

44 Tavia Nyong'o: Afro-Fabulations. The Queer Drama of Black Life, New York 2018, 6.

$45 \mathrm{Im}$ Anschluss an Jared Sexton: "Black life is not lived in the world that the world lives in, but it is lived underground, in outer space." Ders.: The Social Life of Social Death: On AfroPessimism and Black Optimism, in: InTensions Journal, Nr. 5, 2011, 1-47, hier 28.

46 Hartman: Venus in Two Acts, 3 .

47 Ebd., 11.

48 Engemann, Heilmann, Sprenger: Wege und Ziele, 158.
49 Hartman: Venus in Two Acts, 12.

50 Ebd.

51 Wenn ich hier von Repräsentation spreche, dann meine ich, dass sich trotz des poststrukturalistischen Konsenses, Repräsentation als Akt der Herstellung von Wissensgegenständen zu verstehen, Terminologien des Sichtbarmachens, Erkennens und Lückenschließens fortsetzen.

52 Kara Keeling: Queer Times, Black Futures, New York 2019, xv. 